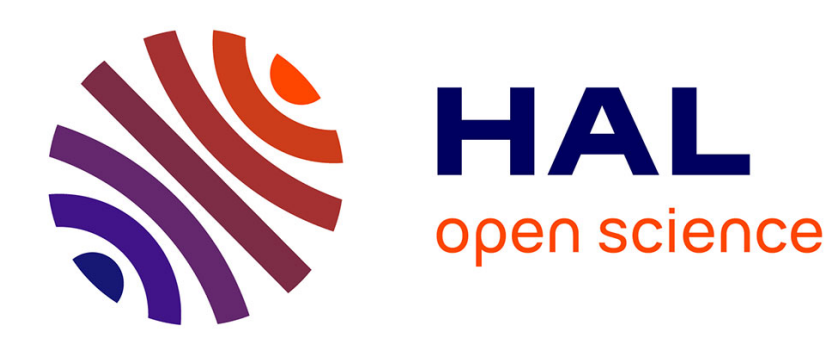

\title{
Orthogonalité des caractères pour GL n sur un corps local de caractéristique non nulle
}

\author{
A. Badulescu
}

\section{To cite this version:}

A. Badulescu. Orthogonalité des caractères pour GL n sur un corps local de caractéristique non nulle. Manuscripta mathematica, 2000, 101 (1), pp.49 - 70. 10.1007/s002290050004 . hal-01822258

\section{HAL Id: hal-01822258 \\ https://hal.science/hal-01822258}

Submitted on 24 Jun 2018

HAL is a multi-disciplinary open access archive for the deposit and dissemination of scientific research documents, whether they are published or not. The documents may come from teaching and research institutions in France or abroad, or from public or private research centers.
L'archive ouverte pluridisciplinaire HAL, est destinée au dépôt et à la diffusion de documents scientifiques de niveau recherche, publiés ou non, émanant des établissements d'enseignement et de recherche français ou étrangers, des laboratoires publics ou privés. 


\title{
Orthogonalité des caractères pour $G L_{n}$ sur un corps local de caractéristique non nulle
}

\author{
Alexandru Ioan BADULESCU \\ Université Paris-Sud, Arithmétique et Géométrie Algébrique, 91405 Orsay \\ E-mail : badulesc@clipper.ens.fr
}

Résumé : On démontre l'orthogonalité des caractères pour $G L_{n}(F)$ où $F$ est un corps local de caractéristique non nulle. On en déduit une correspondance de Jacquet-Langlands avec le groupe des éléments inversibles d'une algèbre à division centrale de dimension $n^{2}$ sur $F$, ainsi que le transfert des intégrales orbitales entre les deux groupes.

\section{Orthogonality relation for characters on the linear group over a local field of positive characteristic}

\begin{abstract}
We prove the orthogonality relations for characters of $G L_{n}(F)$ with $F$ a local field of positive characteristic. Using this, we establish a Jacquet-Langlands correspondence with the group of invertible elements of a central division algebra of dimension $n^{2}$ over $F$, and the transfer of orbital integrals between these two groups.
\end{abstract}

Mots clés : corps local, groupe réductif $p$-adique, représentation.

Code matière MSC : 20G25-20G05.

\section{Introduction}

Une des propriétés générales qu'on espère d'un groupe réductif sur un corps local non archimédien est l'orthogonalité des caractères des représentations de carré intégrable de caractère central fixé. Clozel a montré $([\mathrm{Cl}])$ l'orthogonalité des caractères dans le cas où le corps de base est de caractéristique nulle. Le but principal de cet article est de prouver l'orthogonalité des caractères pour le groupe $G L_{n}(F)$, quand $F$ est un corps local de caractéristique non nulle (sect.4). Cela suffit en particulier pour obtenir le théorème suivant qui étend le résultat de [Ro] à la caractéristique non nulle :

Théorème. Soit $F$ un corps local de caractéristique non nulle et 
$D$ une algèbre à division centrale sur $F$ de dimension $n^{2}$. Alors il existe une unique correspondance bijective entre l'ensemble des classes d'équivalence de représentations essentiellement de carré intégrable irréductibles de $G L_{n}(F)$ et l'ensemble des classes d'équivalence de représentations irréductibles de $D^{*}$ telle que les caractères de deux classes d'équivalence qui se correspondent soient égaux, au signe $(-1)^{n-1}$ près, sur des éléments qui ont le même polynôme caractéristique séparable.

Ce résultat est le premier pas de récurrence dans la démonstration d'une correspondance de Jacquet-Langlands entre $G L_{n}$ et ses formes intérieures en caractéristique non nulle (voir $[\mathrm{DKV}]$ pour la caractéristique nulle), et qui fera le sujet d'un article prochain. Dans la section 5 on montre qu'il y a transfert de toutes les intégrales orbitales de $D^{*}$ vers $G L_{n}(F)$ et de toutes les intégrales orbitales de $G L_{n}(F)$ nulles sur les éléments semisimples réguliers non elliptiques vers $D^{*}$.

La structure de l'article est la suivante : dans la section 2 nous faisons quelques rappels sur les groupes réductifs sur un corps local ; dans la section 3 nous passons en revue quelques résultats de Lemaire concernant les corps proches ; en utilisant ces résultats de Lemaire nous prouvons dans la section 4 le résultat central de cet article, l'orthogonalité des caractères pour $G L_{n}$ sur un corps local de caractéristique non nulle (th.4.4) conséquence, finalement, d'un autre résultat très proche : l'égalité sur l'ensemble des éléments elliptiques séparables entre le conjugué complexe du caractère d'une représentation essentiellement de carré intégrable et l'intégrale orbitale d'un de ses pseudocoefficients (th.4.3); dans la section 5 nous tirons comme conséquence de l'orthogonalité la preuve du théorème énoncé plus haut en remarquant que tous les arguments de la démonstration de [Fl] en caractéristique nulle fonctionnent maintenant en caractéristique non nulle (th.5.1) ; ensuite nous utilisons le résultat du th.5.1 pour en déduire le corollaire 5.2, utile pour la démonstration de la surjectivité de la correspondance entre $G L_{n}$ et toutes ses formes intérieures en caractéristique non nulle (article en préparation, voir [Ba], chap.3) ainsi qu'un résultat de transfert des intégrales orbitales en caractéristique non nulle (cette fois la démonstration de $[\mathrm{Fl}]$ ne fonctionne plus.

\section{Généralités}


Soient $F$ un corps local non archimédien et $G$ le groupe des $F$-points d'un groupe algébrique réductif défini sur $F$. On note $G_{e}$ l'ensemble des éléments elliptiques réguliers de $G$ et $G^{s r}$ l'ensemble des éléments semisimples réguliers de $G$. Soit $Z$ le centre de $G$. Soient $d g$ une mesure de Haar sur $G, d z$ une mesure de Haar sur $Z$ et $d \bar{g}$ la mesure quotient sur $G / Z$. On note $H(G)$ l'espace des fonctions définies sur $G$ à valeurs dans $\mathbb{C}$ qui sont localement constantes et à support compact. Si $K$ est un sous-groupe ouvert compact de $G$ on note $H(G ; K)$ le sous-espace de $H(G)$ formé de fonctions bi-invariantes par $K$.

Soit $\mathcal{K}$ l'ensemble des sous-groupes ouverts compacts de $G$. On a $H(G)=\cup_{K \in \mathcal{K}} H(G ; K)$.

On appelle caractère de $G$ (de $Z$ ) un morphisme continu de groupes de $G($ de $Z)$ dans $\mathbb{C}^{*}$.

Soit $\omega$ un caractère de $Z$. On note $H(G ; \omega)$ l'espace des fonctions définies sur $G$ à valeurs dans $\mathbb{C}$ qui vérifient :

- $f$ est localement constante à support compact modulo $Z$ et

- pour tout $g \in G$ et tout $z \in Z, f(z g)=\omega^{-1}(z) f(g)$

Pour tout $K \in \mathcal{K}$ on note $H(G ; K ; \omega)$ le sous-ensemble de $H(G ; \omega)$ formé de fonctions bi-invariantes par $K$.

On a $H(G ; \omega)=\cup_{K \in \mathcal{K}} H(G ; K ; \omega)$.

Soit $\pi$ une représentation admissible de $G$. Pour tout $f \in H(G)$ on pose $\pi(f)=\int_{G} f(g) \pi(g) d g$. On sait que $\pi(f)$ est alors un opérateur de rang fini, donc à trace. On note $\chi_{\pi}$ la fonction caractère de $\pi$, c'est à dire la fonction définie sur $G^{s r}$ localement constante et qui vérifie, pour tout $f \in H(G)$ qui est à support dans $G^{s r}$,

$$
\operatorname{tr} \pi(f)=\int_{G^{s r}} \chi_{\pi}(g) f(g) d g
$$

(voir [H-C2]). On dit que le caractère de $\pi$ est localement intégrable si $\chi_{\pi}$ est localement intégrable sur $G$ et si la relation plus haut a lieu pour tout $f \in H(G)$, sans condition sur le support. On sait que c'est le cas si $F$ est de caractéristique nulle ([H-C1]) ou si $G=G L_{n}$ ([Le2]).

Soit $\pi$ une représentation admissible de $G$ admettant un caractère central $\omega$ (c'est toujours le cas si $\pi$ est lisse irréductible). Si $f \in$ $H(G ; \omega)$ alors pour tout $z$ dans $Z$ et pour tout $g$ dans $G$ on a $f(z g) \pi(z g)=$ $f(g) \pi(g)$ et on pose $\pi(f)=\int_{G / Z} f(\bar{g}) \pi(\bar{g}) d \bar{g}$.

Pour un caractère $\omega$ fixé on peut définir comme dans $[\mathrm{He}]$ une application de $H(G)$ dans $H(G ; \omega)$ : pour tout $f \in H(G)$ on pose pour tout $g \in G$ :

$$
f_{\omega}(g)=\int_{Z} \omega(z) f(z g) d z
$$


Pour tout tore maximal $T$ de $G$ on fixe une mesure de Haar $d t$ sur $T$. On suppose dans toute la suite que le jeu des mesures ainsi fixées sur les tores maximaux de $G$ vérifie :

- si $T$ et $T^{\prime}$ sont deux tores maximaux de $G$ conjugués dans $G$, les mesures $d t$ et $d t^{\prime}$ fixées sur $T$ et $T^{\prime}$ respectivement se correspondent via cette conjugaison (cela ne dépend pas du choix) et

- si $T$ est un tore maximal elliptique de $G$, alors $d t$ est la mesure de Haar sur $T$ telle que $T / Z$ ait volume égal à 1 pour la mesure quotient $d \bar{t}=d t / d z$.

Par la suite, si $f \in H(G)$ ou $f \in H(G ; \omega)$ pour un certain caractère $\omega$ de $Z$, si $g_{0} \in G^{s r}$, si $T$ (tore maximal) est le commutant de $g_{0}$ dans $G$, on note $\Phi\left(f ; g_{0}\right)=\int_{G / T} f\left(\bar{g}^{-1} g_{0} \bar{g}\right) d \bar{g}$ l'intégrale orbitale de $f$ au point $g$ pour le choix de mesures plus haut.

Le résultat suivant est souvent sous-entendu dans la littérature. Nous donnons la démonstration pour la commodité du lecteur.

Proposition 2.1. . a) Quel que soit $f \in H(G), f_{\omega} \in H(G ; \omega)$. On obtient ainsi une application $I_{\omega}: H(G) \rightarrow H(G ; \omega)$.

b) $I_{\omega}$ est surjective.

c) pour tout $f \in H(G)$ et pour toute représentation admissible $\pi$ de $G$ de caractère central $\omega$, on a $\pi(f)=\pi\left(f_{\omega}\right)$.

d) pour tout $f \in H(G)$, pour tout $g \in G$, on a :

$$
\Phi\left(f_{\omega} ; g\right)=\int_{Z} \omega(z) \Phi(f ; z g) d z .
$$

Démonstration. a) On a $H(G)=\cup_{K \in \mathcal{K}} H(G ; K)$. Pour $K \in \mathcal{K}$ notons abusivement $K \backslash G / K$ un ensemble de représentants des classes d'équivalence dans $K \backslash G / K$. Alors, pour chaque $K \in \mathcal{K}$ l'ensemble $\left\{\mathbf{1}_{K g K}\right\}_{K \backslash G / K}$ est une base du $\mathbb{C}$-espace vectoriel $H(G ; K)$. Soit $g_{0} \in$ $K \backslash G / K$. Un calcul sans difficulté montre qu'on a :

$$
I_{\omega}\left(\mathbf{1}_{K g_{0} K}\right) \equiv 0
$$

si $Z \cap K g_{0} K g_{0}^{-1} k e r(\omega)$

et

$I_{\omega}\left(\mathbf{1}_{K g_{0} K}\right)(g)=\left\{\begin{array}{l}0 \text { si } g \notin Z K g_{0} K \\ \omega^{-1}\left(z_{0}\right) \operatorname{mes}\left(Z \cap K g_{0} K g_{0}^{-1}\right) \text { pour } g \in Z K g_{0} K, g=z_{0} k_{1} g_{0} k_{2}\end{array}\right.$

si $Z \cap K g_{0} K g_{0}^{-1} \subset k e r(\omega)$.

On voit que $I_{\omega}\left(\mathbf{1}_{K g_{0} K}\right)$ vérifie :

- $\operatorname{supp}\left(I_{\omega}\left(\mathbf{1}_{K g_{0} K}\right)\right) \subset Z K g_{0} K$

- $I_{\omega}\left(\mathbf{1}_{K g_{0} K}\right)$ est bi-invariante par $K$

- $I_{\omega}\left(\mathbf{1}_{K g_{0} K}\right)(z g)=\omega^{-1}(z) I_{\omega}\left(\mathbf{1}_{K g_{0} K}\right)(g) \forall z \in Z, \forall g \in G$. 
Ainsi $I_{\omega}\left(\mathbf{1}_{K g_{0} K}\right) \in H(G ; K ; \omega)$ et le point (a) est démontré.

b) Montrons la surjectivité de $I_{\omega}$. Soit $h \in H(G ; \omega)$. Il existe $K$ sous-groupe ouvert et compact de $G$ tel que $h \in H(G ; K ; \omega)$. On va montrer qu'il existe $f \in H(G ; K)$ tel que $I_{\omega}(f)=h$. Si $\alpha \in \operatorname{supp}(h)$, on a par le même calcul immédiat que plus haut

$$
Z \cap K \alpha K \alpha^{-1} \subset \operatorname{ker}(\omega) .
$$

Maintenant $\{K \alpha K\}_{\alpha \in K \backslash G / K}$ est une partition de $G$, et $h$ étant biinvariante par $K$, il existe des $\alpha_{j} \in G, j \in J$, tels que $\left\{K \alpha_{j} K\right\}_{j \in J}$ soit une partition de $\operatorname{supp}(h)$ et que $h$ soit constante et égale à $h\left(\alpha_{j}\right)$ sur $K \alpha_{j} K$ pour tout $j \in J$. De plus, si on connaît $h$ sur $K \alpha_{j} K$, on la connaît sur $z K \alpha_{j} K$ pour tout $z \in Z$ : c'est $\omega^{-1}(z) h\left(\alpha_{j}\right)$. Comme $\operatorname{supp}(h)$ est compact modulo $Z$ on peut trouver un sous-ensemble fini $J^{\prime}$ de $J$ tel que :

$$
h(g)=\left\{\begin{array}{l}
\omega^{-1}(z) h\left(\alpha_{j}\right) \text { si } g \in K z \alpha_{j} K \text { pour un } z \in Z \text { et un } j \in J^{\prime} \\
0 \text { si } g \notin \cup_{j \in J^{\prime}, z \in Z} K z \alpha_{j} K .
\end{array}\right.
$$

Comme on a vu que $\left[\alpha_{j} \in \operatorname{supp}(h)\right] \Rightarrow\left[Z \cap K \alpha_{j} K \alpha_{j}^{-1} \subset \operatorname{ker}(\omega)\right]$, d'après le calcul de $I_{\omega}\left(\mathbf{1}_{K g_{0} K}\right)$ qu'on a fait au début, si on pose

$$
h=\sum_{j \in J^{\prime}} h\left(\alpha_{j}\right)\left[\operatorname{mes}\left(Z \cap K \alpha_{j} K \alpha_{j}^{-1}\right)\right]^{-1} \mathbf{1}_{K \alpha_{j} K},
$$

alors on a bien $I_{\omega}(f)=h$.

c) On a :

$$
\begin{gathered}
\pi\left(f_{\omega}\right)=\int_{G / Z} f_{\omega}(\bar{g}) \pi(\bar{g}) d \bar{g}=\int_{G / Z}\left\{\int_{Z} \omega(z) f(z \bar{g}) d z\right\} \pi(\bar{g}) d \bar{g}= \\
=\int_{G / Z} \int_{Z} \omega(z) f(z \bar{g}) \pi(\bar{g}) d z d \bar{g}=\int_{G / Z} \int_{Z} f(z \bar{g}) \pi(z \bar{g}) d z d \bar{g}= \\
=\int_{G} f(g) \pi(g) d g=\pi(f) .
\end{gathered}
$$

d) Calculons pour $g_{0} \in G^{s r}$ ( $T$ désigne ici le commutant de $g_{0}$ dans $G)$ :

$$
\begin{gathered}
\Phi\left(g_{0} ; f_{\omega}\right)=\int_{G / T} f_{\omega}\left(\bar{g}^{-1} g_{0} \bar{g}\right) d \bar{g}=\int_{G / T}\left\{\int_{Z} \omega(z) f\left(z \bar{g}^{-1} g_{0} \bar{g}\right) d z\right\} d \bar{g}= \\
=\int_{Z} \omega(z)\left\{\int_{G / T} f\left(z \bar{g}^{-1} g_{0} \bar{g}\right) d \bar{g}\right\} d z=\int_{Z} \omega(z) \Phi\left(z g_{0} ; f\right) d z
\end{gathered}
$$


Soient $E(G)$ l'ensemble de classes d'équivalence des représentations lisses irréductibles de $G$ et, pour tout caractère $\omega$ de $Z, E(G ; \omega)$ le sousensemble de $E(G)$ formé de classes d'équivalence de caractère central $\omega$. On note $\Psi(G)$ l'ensemble des caractères non ramifiés de $G$ muni de sa structure usuelle de variété algébrique. Si $\pi \in E(G)$, on note $\Psi(G ; \pi)$ le sous-ensemble de $E(G)$ formé de classes d'équivalence du type $\chi \otimes \pi$ où $\chi \in \Psi(G)$, muni de sa structure de variété algébrique isomorphe à un quotient de $\Psi(G)$. On note $E^{2}(G)$ le sous-ensemble de $E(G)$ formé de classes d'équivalence des représentations essentiellement de carré intégrable et pour tout caractère $\omega$ de $Z$ on note $E^{2}(G ; \omega)$ l'ensemble $E(G ; \omega) \cap E^{2}(G)$. De même, on note $E^{t}(G)$ le sous-ensemble de $E(G)$ formé de classes d'équivalence des représentations essentiellement tempérées et pour tout caractère $\omega$ de $Z$ on note $E^{t}(G ; \omega)$ l'ensemble $E(G ; \omega) \cap E^{t}(G)$.

Fixons un caractère $\omega$ de $Z$. Soit $\pi \in E^{2}(G ; \omega)$. On dit que $f_{\pi} \in$ $H(G ; \omega)$ est un pseudocoefficient de $\pi$ si $\operatorname{tr} \pi\left(f_{\pi}\right)=1$ et $\operatorname{tr} \tau\left(f_{\pi}\right)=0$ pour toute représentation $\tau \in E^{t}(G ; \omega) \backslash\{\pi\}$.

Théorème 2.2. . Soit $\pi \in E^{2}(G ; \omega)$.

a) Alors $\pi$ possède un pseudocoefficient $f_{\pi}$.

b) Pour tout sous-groupe parabolique propre $P$ de $G$, si $L$ est un sous-groupe de Levi de $P$, si $\rho$ est une représentation lisse irréductible de $L$ telle que la restriction du caractère central de $\rho$ à $Z$ est $\omega$, on a $\operatorname{tr}\left(i n d_{P}^{G} \rho\right)\left(f_{\pi}\right)=0$.

c) L'intégrale orbitale sur $G^{s r}$ d'un pseudocoefficient de $\pi$ ne dépend que de $\pi$. Elle est nulle sur $G^{s r} \backslash G_{e}$.

Démonstration. Quitte à tordre par un caractère de $G$, on peut supposer que $\omega$ est unitaire. On démontre le théorème dans ce cas :

a) Soit $A$ l'ensemble fini des caractères $\chi \in \Psi(G)$ tels que le caractère central de $\chi \otimes \pi$ soit $\omega$ (c'est l'ensemble des caractères non ramifiés $\chi$ de $G$ dont la restriction à $Z$ est triviale). Soit $P$ une fonction algébrique définie sur $\Psi(G ; \pi)$ à valeurs dans $\mathbb{C}$ telle que $P(\pi)=1$ et $P(\chi \otimes \pi)=0$ si $\chi \in A, \chi \otimes \pi \neq \pi$. Fixons une paire parabolique minimale de $G$. On sait que l'ensemble des classes d'équivalence de représentations de $G$ du type $i n d_{P}^{G}(\theta \otimes \tau)$ où $P$ est un sous-groupe parabolique standard de $G$, $\tau$ est une représentation tempérée irréductible du sous-groupe de Levi standard $L$ de $P$ et $\theta$ est un caractère strictement positif (relativement à $P$ ) de $L$ forme une base sur $\mathbb{Z}$ du groupe de Grothendieck $\operatorname{Grot}(G)$ de $G$ (voir $[\mathrm{DKV}]$ A.4.f, pour la définition d'un caractère strictement positif et pour la démonstration de ce résultat). On l'appelle ici la base de Langlands de $G \operatorname{rot}(G)$. On appelle les éléments de cette base des représentations standard de $G$ et on dit qu'une représentation standard 
$i n d_{P}^{G}(\theta \otimes \tau)$ est induite stricte si $P$ est un sous-groupe parabolique propre de $G$.

Soit $h: G \operatorname{rot}(G) \rightarrow \mathbb{C}$ l'application linéaire définie par :

- $h(\sigma)=P(\sigma)$ si $\sigma \in \Psi(G ; \pi)$ et

- $h(\sigma)=0$ si $\sigma$ est une représentation de la base de Langlands de $G \operatorname{rot}(G)$ qui ne se trouve pas dans $\Psi(G ; \pi)$.

On vérifie que $h$ est une bonne fonction au sens de [BDK] 1.2. Le clou de la démonstration est le lemme suivant :

Lemme 2.3. . Soit $(A ; P)$ une paire parabolique standard de $G$ et $P=$ $L U$ la décomposition de Levi standard de P. Soit $\tau \in E^{t}(L)$. Écrivons $\tau=\theta \otimes \tau_{0}$ où $\theta$ est un caractère réel de $L$ et $\tau_{0}$ une représentation tempérée irréductible de L. Alors l'image de ind ${ }_{P}^{G} \tau$ dans $\operatorname{Grot}(G)$ est

(i) une somme de représentations essentiellement tempérées de $G$ s'il existe un caractère $\Theta$ de $G$ tel que res ${ }_{L}^{G} \Theta=\theta$ et

(ii) une somme de représentations standard induites strictes sinon.

Démonstration. Ce lemme se réduit au fait suivant, facile à démontrer : si le caractère (réel) $\theta$ n'est pas trivial, alors il existe un triplet $\left(L^{\prime} ; \theta^{\prime} ; g\right)$ tel que $L^{\prime} \neq G, L \subset L^{\prime}, \theta^{\prime}$ est un caractère de $L^{\prime}$ tel que $\operatorname{res}_{L}^{L^{\prime}} \theta^{\prime}=\theta, A d(g) L^{\prime}$ est standard et $A d(g) \theta^{\prime}$ est un caractère strictement positif de $A d(g) L^{\prime}$ (relativement au sous-groupe parabolique standard de $G$ associé à $\left.A d(g) L^{\prime}\right)$.

Ce lemme implique que notre fonction $h$ s'annule sur toute représentation de $G$ qui est induite à partir d'une représentation lisse irréductible d'un sous-groupe parabolique propre (parce que, si $L$ est un sous-groupe de Levi de $G$, l'ensemble des induites des représentations essentiellement tempérées de tous les sous-groupes de Levi de $L$ à $L$ est une famille génératrice dans $\operatorname{Grot}(L))$. Pour vérifier alors que $h$ est une bonne fonction, il suffit de vérifier que $h$ est algébrique sur $\Psi(G ; \sigma)$ pour toute $\sigma \in E(G)$. En décomposant $\sigma$ sur la base de Langlands, on voit qu'il suffit de le prouver dans le cas où $\sigma \in E^{t}(G)$. Or, pour une telle représentation $\sigma$, tout élément de $\Psi(G ; \sigma)$ est élément de la base de Langlands donc, par construction, $h$ est algébrique sur $\Psi(G ; \sigma)$ si $\sigma \in \Psi(G ; \pi)$ et nulle sur $\Psi(G ; \sigma)$ sinon. Donc $h$ est une bonne fonction.

On applique le théorème de Paley-Wiener ([DKV] 1.2) : soit $f \in$ $H(G)$ telle que pour tout $\sigma \in E(G)$ on ait $\operatorname{tr} \sigma(f)=h(\sigma)$. Posons $f_{\pi}=I_{\omega}(f) \in H(G ; \omega)$. La fonction $f_{\pi}$ ainsi définie vérifie clairement les conditions voulues, puisque pour tout $\sigma \in E(G ; \omega)$ on a $\operatorname{tr} \sigma\left(f_{\pi}\right)=\operatorname{tr} \sigma(f)$. 
b) Le pseudocoefficient $f_{\pi}$ de $\pi$ construit au point a) vérifie cette propriété puisque pour toute $\sigma \in E(G ; \omega)$ on a $\operatorname{tr} \sigma\left(f_{\pi}\right)=\operatorname{tr} \sigma(f)$ et que $f$ annule la trace de toute représentation induite à partir d'un sous-groupe parabolique propre. Il suffit donc de montrer que, si $f_{\pi}^{\prime}$ est un autre pseudocoefficient de $\pi$, alors $\operatorname{tr} \sigma\left(f_{\pi}-f_{\pi}^{\prime}\right)=0$ pour tout $\sigma \in$ $E(G ; \omega)$. Soit $\sigma \in E(G ; \omega)$; décomposons $\sigma$ sur la base de Langlands $\operatorname{de} \operatorname{Grot}(G)$ :

$$
\sigma=\sum_{i=1}^{n} a_{i} \pi_{i} .
$$

Pour chaque $\pi_{i}$ il existe un sous-groupe parabolique standard $P_{i} \operatorname{de} G$ et une représentation irréductible essentiellement tempérée $\tau_{i}$ du sousgroupe de Levi standard $L_{i}$ de $P_{i}$ telle que $\pi=i n d_{P_{i}}^{G} \tau_{i}$. On remarque que pour tout $i, \pi_{i}$ admet un caractère central, restriction du caractère central de $\tau_{i}$ à $Z$. Par indépendance linéaire des distributions caractères on trouve alors que pour tout $i$, la restriction du caractère central de $\tau_{i}$ à $Z$ est $\omega$. Alors $\operatorname{tr}\left(i n d_{P_{i}}^{G} \tau_{i}\right)\left(f_{\pi}-f_{\pi}^{\prime}\right)=0$ par le fait que les caractères dans $\Psi\left(L_{i}\right)$ unitaires et dont la restriction à $Z$ est $\omega$ forment un ensemble Zariski dense dans la sous-variété de $\Psi\left(L_{i}\right)$ formée des caractères dans $\Psi\left(L_{i}\right)$ dont la restriction à $Z$ est $\omega$.

c) On a montré que deux pseudocoefficients de $\pi$ ont la même trace sur les éléments de $E(G ; \omega)$. Par [DKV], th.A.2.a, variante 2 (valable en toute caractéristique), ils ont la même intégrale orbitale sur $G^{s r}$. Pour montrer que l'intégrale orbitale d'un pseudocoefficient de $\pi$ s'annule sur $G^{s r} \backslash G_{e}$ il suffit donc de le montrer pour un pseudocoefficient particulier. On le vérifie pour le pseudocoefficient $f_{\pi}$ construit au point a). On avait $f_{\pi}=I_{\omega}(f)$, où $f$ était un certain élément de $H(G)$ dont on sait qu'il annule la trace de toute représentation induite à partir d'un sous-groupe parabolique propre de $G$.

Lemme 2.4. L'intégrale orbitale de $f$ est nulle sur $G^{s r} \backslash G_{e}$.

Démonstration. Soit $g \in G^{s r} \backslash G_{e}$ et soient $L$ un sous-groupe de Levi propre de $G$ tel que $g \in L$ et $P$ un sous-groupe parabolique (propre) de $G$ tel que $L$ soit un sous-groupe de Levi de $P$. Si on note $f^{P}$ le terme constant de $f$ le long de $P$, alors pour toute représentation $\sigma$ de $L$ on a :

$$
\operatorname{tr} \sigma\left(f^{P}\right)=\operatorname{tr} i n d_{P}^{G} \sigma(f)=0
$$

où pour la première égalité on a utilisé [La], lemme 7.5.7. On en déduit que l'intégrale orbitale de $f^{P}$ est nulle sur les éléments semisimples réguliers de $L$ par $[\mathrm{DKV}]$, th.A.2.a, et finalement, comme $\Phi(f ; g)=$ $\Phi\left(f^{P} ; g\right)([\mathrm{La}]$, prop.4.3.11), que $\Phi(f ; g)=0$. 
Ce lemme et la proposition ??.d impliquent que l'intégrale orbitale de $I_{\omega}(f)=f_{\pi}$ s'annule aussi sur $G^{s r} \backslash G_{e}$.

Avec les notations et conventions fixées plus haut (notamment les mesures sur les tores elliptiques), on dit que $G$ a la propriété $\mathbf{P}$ si :

pour toute représentation de carré intégrable $\pi$ de $G$ de caractère central (unitaire) $\omega$, si on note $f_{\pi}$ un pseudocoefficient de $\pi$ dans $H(G ; \omega)$, alors

(i) l'intégrale orbitale de $f_{\pi}$ est nulle sur les éléments semisimples réguliers non elliptiques et

(ii) l'intégrale orbitale de $f_{\pi}$ est égale au conjugué complexe de $\chi_{\pi}$ sur l'ensemble des éléments elliptiques réguliers de $G$.

On a vu (th.??) que le point (i) est toujours vrai. On sait par ailleurs (démonstration de la prop.6, $[\mathrm{Cl}]$ ) que, si $F$ est de caractéristique nulle, le point (ii) est vrai aussi.

Soit $\mathcal{T}$ un ensemble de représentants des classes de conjugaison des tores maximaux de $G$. Pour tout tore $T \in \mathcal{T}$ on note $T^{\text {reg }}$ l'ensemble des éléments réguliers de $T, W_{T}$ le groupe de Weyl de $T$ et $\left|W_{T}\right|$ le cardinal de ce groupe. Pour tout élément semisimple régulier $g$ de $G$ de centralisateur $T$, on note $D(g)$ la valeur absolue normalisée de $\operatorname{det}\left(A d\left(g^{-1}\right)-1\right)$ agissant sur Lie $(G) / \operatorname{Lie}(T)$. Pour tout caractère unitaire $\omega$ de $Z$ on note $L^{0}\left(G_{e} ; \omega\right)$ l'espace des fonctions $f$ définies sur $G_{e}$ à valeurs dans $\mathbb{C}$ qui sont localement constantes, invariantes par conjugaison par des éléments de $G$, et vérifient $f(z g)=\omega(z) f(g)$ pour tout $g \in G$ et tout $z \in Z$. Soit $\mathcal{T}_{e}$ le sous-ensemble de $\mathcal{T}$ formé des représentants des classes de conjugaison de tores maximaux elliptiques. On note $L^{2}\left(G_{e} ; \omega\right)$ le sous-espace de $L^{0}\left(G_{e} ; \omega\right)$ formé des fonctions $f$ pour lesquelles

$$
\sum_{T \in \mathcal{T}_{e}}\left|W_{T}\right|^{-1} \int_{T^{r e g} / Z} D(t)|f(t)|^{2} d \bar{t}
$$

converge. On définit un produit scalaire dans $L^{2}\left(G_{e} ; \omega\right)$ en posant :

$$
<f_{1} ; f_{2}>_{e}=\sum_{T}\left|W_{T}\right|^{-1} \int_{T^{r e g} / Z} D(t) f_{1}(t) \overline{f_{2}(t)} d \bar{t}
$$

ce qui donne à $L^{2}\left(G_{e} ; \omega\right)$ une structure d'espace préhilbertien.

On dit qu'il y a orthogonalité des caractères sur $G$ si pour tout caractère unitaire $\omega$ de $Z$, pour tout $\pi \in E^{2}(G ; \omega)$, la restriction de $\chi_{\pi}$ à 
$G_{e}$ se trouve dans $L^{2}\left(G_{e} ; \omega\right)$ et qu'on a, pour tout $\pi, \pi^{\prime}$ dans $E^{2}(G ; \omega)$,

$$
<\chi_{\pi} ; \chi_{\pi^{\prime}}>=\left\{\begin{array}{l}
0 \text { si } \pi \neq \pi^{\prime} \\
1 \text { si } \pi=\pi^{\prime}
\end{array} .\right.
$$

Si le corps de base $F$ est de caractéristique nulle, on sait ([Cl], th.3) qu'il y a toujours orthogonalité des caractères sur $G$. Dans [DKV], partie A.3.h, on montre la proposition suivante (indépendamment de la caractéristique de $F$ ) :

Proposition 2.5. . Si G est tel que :

a) pour tout $\pi \in E^{2}(G)$, le caractère de $\pi$ est localement intégrable et

b) G a la propriété $\mathbf{P}$, alors il y a orthogonalité des caractères sur $G$.

\section{Corps locaux proches et le groupe linéaire. Les résultats de Lemaire}

Soient $F$ un corps local de caractéristique non nulle et $n$ un entier strictement positif, qu'on considère fixés par la suite. On note $O_{F}$ l'anneau des entiers de $F, P_{F}$ l'idéal maximal de $O_{F}$ et on fixe une uniformisante $\pi_{F}$ de $F$. On note $K_{F}$ le sous-groupe compact maximal $G L_{n}\left(O_{F}\right)$ de $G L_{n}(F)$ et pour tout $p \in \mathbb{N}^{*}$ on note $K_{F}^{p}$ le sous-groupe de congruence de niveau $p$ de $G L_{n}(F)$ qui est par définition le noyau de la projection $K_{F} \rightarrow G L_{n}\left(O_{F} / P_{F}^{p}\right)$. On note $H\left(G L_{n}(F) ; K_{F}^{p}\right)$ la sous-algèbre de Hecke de $H\left(G L_{n}(F)\right)$ formée des fonctions qui sont bi-invariantes par $K_{F}^{p}$. Pour un tel corps $F$ on considère d'une façon générale : sur le centre $Z$ de $G L_{n}(F)$ identifié à $F^{*}$ la mesure de Haar $d z$ pour laquelle $O_{F}^{*}$ est de volume égal à 1 , sur $G L_{n}(F)$ la mesure de Haar $d g$ pour laquelle $K_{F}$ est de volume égal à 1 et sur tout tore maximal elliptique $T$ la mesure de Haar $d t$ telle que $T / Z$ soit de volume égal à 1 pour la mesure quotient $d t / d z$. Si $g$ est un élément elliptique régulier de $G L_{n}(F)$ et $f \in H\left(G L_{n}(F)\right)$ ou $f \in H\left(G L_{n}(F) ; \omega\right)$ pour un caractère $\omega$ de $Z, \Phi(f ; g)$ désigne l'intégrale orbitale de $f$ au point $g$ pour les mesures ainsi choisies. Si $\pi$ est une représentation de $G L_{n}(F)$ on appelle niveau de $\pi$ (notation niv $(\pi)$ ) le plus petit entier $m$ tel que $\pi$ ait un vecteur fixe sous $K_{F}^{m}$, avec la convention $K_{F}^{0}=K_{F}$. Le niveau de $\pi$ est aussi le plus petit entier $m$ tel que $\pi$ induise une représentation non identiquement nulle de $H\left(G L_{n}(F) ; K_{F}^{m}\right)$. 
On note $B_{F}$ le sous-groupe de $G L_{n}(F)$ formé des matrices dans $G L_{n}\left(O_{F}\right)$ qui sont triangulaires supérieures modulo $P_{F}$. On pose aussi, pour tout entier strictement positif $m, B_{F}^{m}=1+\pi_{F}^{m} B_{F}$ et par convention $B_{F}=B_{F}^{0}$ et on note $H\left(G L_{n}(F) ; B_{F}^{m}\right)$ l'espace des fonctions localement constantes à support compact sur $G L_{n}(F)$ qui sont bi-invariantes par $B_{F}^{m}$. Pour tout entier positif $m$ on a :

$$
K_{F}^{m+1} \subset B_{F}^{m} \subset K_{F}^{m} .
$$

Soit maintenant $L$ un corps local non archimédien de caractéristique nulle. On adopte les mêmes notations pour $L$. Soit $m$ un entier strictement positif. On dit que $F$ et $L$ sont $m$-proches s'il y a un isomorphisme d'anneaux $O_{F} / P_{F}^{m} \simeq O_{L} / P_{L}^{m}$. On fixe alors un triplet $\left(\bar{\lambda}_{F L}^{m} ; \pi_{F} ; \pi_{L}\right)$ où $\bar{\lambda}_{F L}^{m}: O_{F} / P_{F}^{m} \rightarrow O_{L} / P_{L}^{m}$ est un isomorphisme et $\pi_{L}$ est une uniformisante de $L$ telle que $\bar{\lambda}_{F L}^{m}$ (classe de $\left.\pi_{F}\right)=$ classe de $\pi_{L}$.

Si $L$ est un corps local non archimédien de caractéristique nulle tel que $F$ et $L$ soient $m$-proches alors $\bar{\lambda}_{F L}^{m}$ induit un isomorphisme d'espaces vectoriels de $H\left(G L_{n}(F) ; B_{F}^{m}\right)$ sur $H\left(G L_{n}(L) ; B_{L}^{m}\right)$, isomorphisme dépendant du choix de $\pi_{F}$ et $\pi_{L}$ (fixés dans la suite). Cet isomorphisme d'espaces vectoriels est en fait un isomorphisme d'algèbres (proposition 2.1.1, [Le1]). La restriction de cet isomorphisme à la sousalgèbre $H\left(G L_{n}(F) ; K_{F}^{m}\right)$ de $H\left(G L_{n}(F) ; B_{F}^{m}\right)$ induit un isomorphisme d'algèbres :

$$
\bar{\zeta}_{F L}^{m}: H\left(G L_{n}(F) ; K_{F}^{m}\right) \rightarrow H\left(G L_{n}(L) ; K_{L}^{m}\right)
$$

(proposition 2.2.1 [Le1]). En tant qu'isomorphisme d'espaces vectoriels, $\bar{\zeta}_{F L}^{m}$ est défini de la façon suivante :

Posons

$$
\mathcal{A}_{F}=\left\{A=\left(a_{i j}\right)_{i, j} \in G L_{n}(F): a_{i j}=\delta_{i j} \pi_{F}^{a_{i}}, a_{1} \leq a_{2} \leq \ldots \leq a_{n}\right\}
$$

où $\delta_{i j}$ est le symbole de Kroneker. On a alors

$$
G L_{n}(F)=\coprod_{A \in \mathcal{A}_{F}} K_{F} A K_{F}
$$

Posons

$$
\mathbf{T}_{F, m}=G L_{n}\left(O_{F} / P_{F}^{m}\right) \times G L_{n}\left(O_{F} / P_{F}^{m}\right) .
$$

Soit $A \in \mathcal{A}_{F}$. Si $(B ; C) \in K_{F} \times K_{F}$, alors $K_{F}^{m} B A C^{-1} K_{F}^{m}$ ne dépend que de la classe $(\hat{B} ; \hat{C})$ de $(B ; C)$ dans $\mathbf{T}_{F, m}$. On peut donc définir sans ambiguité $K_{F}^{m} \hat{B} A \hat{C}^{-1} K_{F}^{m}$ pour tout $(\hat{B} ; \hat{C}) \in \mathbf{T}_{F, m}$. Notons maintenant $\mathbf{H}_{F, m, A}$ l'ensemble des couples $(\hat{B} ; \hat{C}) \in \mathbf{T}_{F, m}$ tels qu'il existe un représentant $B$ de $\hat{B}$ dans $G L_{n}\left(O_{F}\right)$ et un représentant $C$ de $\hat{C}$ dans $G L_{n}\left(O_{F}\right)$ tel qu'on ait $B A=A C$. En écrivant cette relation 
$B A C^{-1}=A$ on vérifie que $\mathbf{H}_{F, m, A}$ est un sous-groupe de $\mathbf{T}_{F, m}$. Posons enfin

$$
\tilde{\mathbf{T}}_{F, m, A}=\mathbf{T}_{F, m} / \mathbf{H}_{F, m, A}
$$

Un simple calcul montre que

$$
K_{F} A K_{F}=\coprod_{(\tilde{B} ; \tilde{C}) \in \tilde{\mathbf{T}}_{F, m, A}} K_{F}^{m} \tilde{B} A \tilde{C}^{-1} K_{F}^{m}
$$

Finalement on a obtenu la décomposition

$$
\left(\mathbf{D}_{\mathbf{m}}\right) \quad G L_{n}(F)=\coprod_{A \in \mathcal{A}_{F}} \coprod_{(\tilde{B} ; \tilde{C}) \in \tilde{\mathbf{T}}_{F, m, A}} K_{F}^{m} \tilde{B} A \tilde{C}^{-1} K_{F}^{m}
$$

On en déduit que l'ensemble des fonctions caractéristiques des ensembles compacts $K_{F}^{m} \tilde{B} A \tilde{C}^{-1} K_{F}^{m}$, pour tout $(A ;(\tilde{B} ; \tilde{C})), A \in \mathcal{A}_{F}$ et $(\tilde{B} ; \tilde{C}) \in \tilde{\mathbf{T}}_{F, m, A}$, est une base algébrique de $H\left(G L_{n}(F) ; K_{F}^{m}\right)$. On raisonne de la même façon pour $G L_{n}(L)$, en adoptant les notations analogues. Maintenant il y a une bijection $\zeta_{F L}: \mathcal{A}_{F} \simeq \mathcal{A}_{L}$ qui envoie $\operatorname{diag}\left(\pi_{F}^{a_{1}} ; \pi_{F}^{a_{2}} ; \ldots \pi_{F}^{a_{n}}\right)$ sur $\operatorname{diag}\left(\pi_{L}^{a_{1}} ; \pi_{L}^{a_{2}} ; \ldots \pi_{L}^{a_{n}}\right)$, et pour tout $A \in$ $\mathcal{A}_{F}$ une bijection naturelle $\zeta_{F L}^{m}: \tilde{\mathbf{T}}_{F, m, A} \simeq \tilde{\mathbf{T}}_{L, m, \zeta_{F L}(A)}$ induite par l'isomorphisme $G L_{n}\left(O_{F} / P_{F}^{m}\right) \times G L_{n}\left(O_{F} / P_{F}^{m}\right) \simeq G L_{n}\left(O_{L} / P_{L}^{m}\right) \times G L_{n}\left(O_{L} / P_{L}^{m}\right)$. L'isomorphisme $\bar{\zeta}_{F L}^{m}$ est donné par

$$
\bar{\zeta}_{F L}^{m}\left(\mathbf{1}_{K_{F}^{m} \tilde{B} A \tilde{C}^{-1} K_{F}^{m}}\right)=\mathbf{1}_{K_{F}^{m} \tilde{B}^{\prime} \zeta_{F L}(A) \tilde{C}^{\prime}-1 K_{F}^{m}}
$$

pour tout $A \in \mathcal{A}_{F}$ et $(\tilde{B} ; \tilde{C}) \in \tilde{\mathbf{T}}_{F, m, A}$, où $\left(\tilde{B}^{\prime} ; \tilde{C}^{\prime}\right)=\zeta_{F L}^{m}((\tilde{B} ; \tilde{C}))$.

Si $X$ est une partie compacte de $G L_{n}(F)$ bi-invariante par $K_{F}^{m}$, l'image par $\bar{\zeta}_{F L}^{m}$ de la fonction caractéristique de $X$ est la fonction caractéristique d'un sous-ensemble compact $Y$ de $G L_{n}(L)$ bi-invariant par $K_{L}^{m}$ et on pose par définition $\bar{\zeta}_{F L}^{m}(X)=Y$. On a alors $\operatorname{vol}(X)=\operatorname{vol}(Y)$ si les mesures sur $G L_{n}(F)$ et $G L_{n}(L)$ sont comme plus haut.

Soit maintenant $\pi$ une représentation irréductible de niveau inférieur ou égal à $m$ de $G L_{n}(F)$ et notons $V_{\pi}$ l'espace de la représentation $\pi$. Alors l'algèbre $H\left(G L_{n}(F) ; K_{F}^{m}\right)$ agit sur l'espace $V_{\pi}^{K_{F}^{m}}$ des vecteurs fixes sous $K_{\pi}^{m}$ par

$$
f(v)=\pi(f)(v)
$$

pour tout $f \in H\left(G L_{n}(F) ; K_{F}^{m}\right)$ et tout $v \in V_{\pi}^{K_{F}^{m}}$. L'espace $V_{\pi}^{K_{F}^{m}}$ est ainsi muni d'une structure de $H\left(G L_{n}(F) ; K_{F}^{m}\right)$-module à gauche. On note $V_{\pi, H, m}$ le $H\left(G L_{n}(F) ; K_{F}^{m}\right)$-module $V_{\pi}^{K_{F}^{m}}$ pour le différentier du C-espace $V_{\pi}^{K_{F}^{m}}$ avec lequel il coïncide ensemblistement. On sait que $V_{\pi, H, m}$ est un $H\left(G L_{n}(F) ; K_{F}^{m}\right)$-module non nul irréductible et que $\pi \mapsto$ $V_{\pi, H, m}$ induit une bijection entre l'ensemble de classes d'équivalence de représentations irréductibles de $G L_{n}(F)$ de niveau inférieur ou égal à 
$m$ et l'ensemble des classes d'isomorphie de $H\left(G L_{n}(F) ; K_{F}^{m}\right)$-modules irréductibles. Maintenant, l'isomorphisme $\bar{\zeta}_{F L}^{m}: H\left(G L_{n}(F) ; K_{F}^{m}\right) \simeq$ $H\left(G L_{n}(L) ; K_{L}^{m}\right)$ induit un isomorphisme (noté toujours $\bar{\zeta}_{F L}^{m}$ ) entre l'ensemble des classes d'isomorphie de $H\left(G L_{n}(F) ; K_{F}^{m}\right)$-modules irréductibles et l'ensemble des classes d'isomorphie de $H\left(G L_{n}(L) ; K_{L}^{m}\right)$-modules irréductibles. Donc l'image par $\bar{\zeta}_{F L}^{m}$ de la classe d'isomorphie de $V_{\pi, H, m}$ est une classe d'isomorphie de $H\left(G L_{n}(L) ; K_{L}^{m}\right)$-modules irréductibles et elle correspond à une classe d'équivalence $C_{L}$ de représentations irréductibles de niveau inférieur ou égal à $m$ de $G L_{n}(L)$. Si $C_{F}$ est la classe d'équivalence de la représentation $\pi$, on pose $\bar{\zeta}_{F L}^{m}\left(C_{F}\right)=C_{L}$. Par conséquent, $\bar{\zeta}_{F L}^{m}$ induit une bijection de l'ensemble des classes d'équivalence de représentations lisses irréductibles de $G L_{n}(F)$ de niveau inférieur ou égal à $m$ sur l'ensemble des classes d'équivalence de représentations lisses irréductibles de $G L_{n}(L)$ de niveau inférieur ou égal à $m$ - bijection qu'on va toujours noter $\bar{\zeta}_{F L}^{m}$ - et pour toute représentation $\pi$ de niveau inférieur ou égal à $m$ de $G L_{n}(F)$, pour tout $f \in H\left(G L_{n}(F) ; K_{F}^{m}\right)$, on a

$$
\operatorname{tr} \bar{\zeta}_{F L}^{m}(\pi)\left(\bar{\zeta}_{F L}^{m}(f)\right)=\operatorname{tr} \pi(f) .
$$

Remarquons que, si $\sigma \in C_{L}$, il y a par construction isomorphisme de $\mathbb{C}$-espaces vectoriels entre $V_{\pi}^{K_{F}^{m}}$ et $V_{\sigma}^{K_{L}^{m}}$, puisque l'isomorphisme $\bar{\zeta}_{F L}^{m}: H\left(G L_{n}(F) ; K_{F}^{m}\right) \simeq H\left(G L_{n}(L) ; K_{L}^{m}\right)$ est un isomorphisme de $\mathbb{C}$ algèbres.

De plus, l'isomorphisme

$$
\bar{\zeta}_{F L}^{m}: H\left(G L_{n}(F) ; K_{F}^{m}\right) \simeq H\left(G L_{n}(L) ; K_{L}^{m}\right)
$$

induit par restriction un isomorphisme $H\left(G L_{n}(F) ; K_{F}^{m-1}\right) \simeq H\left(G L_{n}(L) ; K_{L}^{m-1}\right)$ qui n'est autre que l'isomorphisme $\bar{\zeta}_{F L}^{m-1}$ construit à partir du triplet $\left(\bar{\lambda}_{F L}^{m-1} ; \pi_{F} ; \pi_{L}\right)$ où $\bar{\lambda}_{F L}^{m-1}: O_{F} / P_{F}^{m-1} \simeq O_{L} / P_{L}^{m-1}$ est l'isomorphisme induit par de $\bar{\lambda}_{F L}^{m}: O_{F} / P_{F}^{m} \simeq O_{L} / P_{L}^{m}$ (parce que les décompositions $\left(D_{m}\right)$ et $\left(D_{m-1}\right)$ de $G L_{n}(F)$ sont compatibles). Cela implique que $\bar{\zeta}_{F L}^{m}(\pi)$ a le même niveau que $\pi$.

Proposition 3.1. . Si $m \geq 2, \bar{\zeta}_{F L}^{m}$ réalise une bijection entre les classes d'équivalence des représentations génériques irréductibles de $G L_{n}(F)$ de niveau inférieur ou égal à $m-1$ et les classes d'équivalence des représentations génériques irréductibles de $G L_{n}(L)$ de niveau inférieur ou égal à $m-1$.

Démonstration. On a expliqué plus haut que la bijection $\bar{\zeta}_{F L}^{m}$ est la restriction aux représentations de $G L_{n}(F)$ de niveau inférieur ou égal à $m$ de la bijection $\zeta$ introduite au chapitre 1 de [Le1]. On applique donc le corollaire 3.3.3 de [Le1] tenant compte du fait que si une représentation $\pi$ a un vecteur $v$ fixe sous $K_{F}^{m-1} v$ est aussi un vecteur 
fixe de $\pi$ sous $B_{F}^{m-1}$.

On va maintenant rappeler un autre résultat de Lemaire qui concerne le comportement des intégrales orbitales elliptiques des fonctions se correspondant sur des corps proches.

Soit $g$ un élément elliptique régulier de $G L_{n}(F)$. Soient :

- $F_{g}=F[g]$ le sous-corps maximal de $M_{n}(F)$ engendré par $g$,

- $e$ l'indice de ramification de l'extension (de degré $n$ ) de corps locaux non archimédiens $F_{g} / F$,

- $\mathcal{G}$ l'unique $O_{F}$-ordre héréditaire de $M_{n}(F)$ normalisé par $F_{g}^{*}$,

- $J_{\mathcal{G}}$ le radical de Jacobson de $\mathcal{G}$, $\left.J_{\mathcal{G}}^{i}\right\}$,

- $\nu_{\mathcal{G}}$ la valuation sur $M_{n}(F)$ définie par $\nu_{\mathcal{G}}(x)=\max \{i \in \mathbb{Z}: x \in$

- pour tout $k \in \mathbb{Z}, \mathcal{N}_{k}=\left\{x \in \mathcal{G}: x g-g x \in J_{\mathcal{G}}^{k}\right\}$,

- $k_{0}=\max \left\{k \in \mathbb{Z}: \mathcal{N}_{k} \not \subset O_{F_{g}}+J_{\mathcal{G}}\right\}$,

$-k_{1}=k_{0}-\nu_{\mathcal{G}}(g)$

- $\bar{k}_{1}=k_{1} / e$,

- $A=P_{F}^{1-k_{1}} \mathcal{N}_{k_{0}}$.

Fixons un caractère additif $\psi_{F}$ de $F$ de conducteur $P_{F}$ (trivial sur $P_{F}$ et non trivial sur $O_{F}$ ). Soit

$$
\Lambda=\left\{x \in M_{n}(F): \operatorname{tr}(x y) \in \operatorname{ker}\left(\psi_{F}\right), \forall y \in A\right\}
$$

Soit $m$ un entier strictement positif. Soient :

- $m^{\prime}=m^{\prime}(g ; m)$ le plus petit entier supérieur ou égal à $\max \{1+$ $\left.\bar{k}_{1}, m-\bar{k}_{1}\right\}$,

- $m^{\prime \prime}=m^{\prime \prime}(g ; m)$ le plus petit entier supérieur ou égal à $m^{\prime}(g ; m)+$ $\bar{k}_{1}+1$.

Théorème 3.2. . Soit $g$ un élément elliptique régulier de $G L_{n}(F)$ et $m$ un entier strictement positif. Alors le voisinage $V(g ; m)$ de $g$ défini par $V(g ; m)=g\left(1+\pi_{F}^{m^{\prime}(g ; m)} \Lambda\right)$ est contenu dans l'ensemble des éléments elliptiques réguliers de $G L_{n}(F)$ et vérifie

a) $V(g ; m)$ est bi-invariant par $K_{F}^{m^{\prime \prime}}$ et pour toute fonction $f \in$ $H\left(G L_{n}(F) ; K_{F}^{m}\right)$ l'intégrale orbitale de $f$ est constante égale à $\Phi(f ; g)$ sur $V(g ; m)$;

b) pour tout corps $L m^{\prime \prime}$-proche de $F, \bar{\zeta}_{F L}^{m^{\prime \prime}}(V(g ; m))$ est incluse dans l'ensemble des éléments elliptiques réguliers de $G L_{n}(L)$ et pour toute fonction $f \in H\left(G L_{n}(F) ; K_{F}^{m}\right)$, l'intégrale orbitale de $\bar{\zeta}_{F L}^{m^{\prime \prime}}(f)$ est constante égale à $\Phi(f ; g)$ sur $\bar{\zeta}_{F L}^{m^{\prime \prime}}(V(g ; m))$. 
Démonstration. a) On a $K_{F}^{m^{\prime \prime}} \subset B_{F}^{m^{\prime \prime}-1}$ et on applique le théorème de la page 1042 de [Le3].

b) On a

$$
f \in H\left(G L_{n}(F) ; K_{F}^{m}\right) \Rightarrow f \in H\left(G L_{n}(F) ; B_{F}^{m}\right) .
$$

On applique à nouveau le théorème de la page 1042 de [Le3].

\section{Orthogonalité des caractères sur $G L_{n}(F)$ en caractéristique non nulle}

On a besoin d'une variante du théorème ?? qui concerne les fonctions de $H\left(G L_{n}(F) ; \omega\right)$ où $\omega$ est un caractère unitaire fixé du centre $Z_{F}$ de $G L_{n}(F)$. Tout d'abord un tel caractère est une représentation lisse et irréductible de $G L_{1}(F)$ et par conséquent, s'il est de conducteur $1+P_{F}^{m}$ alors pour tout corps $L m$-proche de $F$ on sait définir $\bar{\zeta}_{F L}^{m}(\omega)$ qui est un caractère de $G L_{1}(L)$ qu'on identifiera au centre $Z_{L}$ de $G L_{n}(L)$. Si $\pi$ est une représentation lisse irréductible de $G L_{n}(F)$ de niveau $m$ et de caractère central $\omega$ alors $\omega$ est trivial sur $1+P_{F}^{m}$ et pour tout corps $L m$-proche de $F$ le caractère central de $\bar{\zeta}_{F L}^{m}(\pi)$ est $\bar{\zeta}_{F L}^{m}(\omega)$. Si $\omega$ est un tel caractère de $F^{*}$ de niveau inférieur ou égal à $m$ et si $f \in H\left(G L_{n}(F) ; K_{F}^{m}\right)$, alors on a, avec les notations de la prop.?? :

Théorème 4.1. . Si g est un élément elliptique régulier de $G L_{n}(F)$ et $V(g ; m)$ et $m^{\prime \prime}(g ; m)$ sont comme plus haut, alors l'intégrale orbitale de $I_{\omega}(f)$ est constante égale à $\Phi\left(I_{\omega}(f) ; g\right)$ sur $V(g ; m)$ et pour tout corps local $L$ qui est $m^{\prime \prime}(g ; m)$-proche de $F$, l'intégrale orbitale de $I_{\bar{\zeta}_{F L}^{m \prime \prime}(\omega)}\left(\bar{\zeta}_{F L}^{m^{\prime \prime}}(f)\right)$ est constante et égale à $\Phi\left(I_{\omega}(f) ; g\right)$ sur $\bar{\zeta}_{F L}^{m^{\prime \prime}}(V(g ; m))$.

Démonstration. On a (prop.??.d) la relation

$$
\Phi\left(I_{\omega}(f) ; g\right)=\int_{Z} \omega(z) \Phi(f ; z g) d z .
$$

Montrons que pour tout $z \in Z$ nous avons $V(z g ; m)=z V(g ; m)$ et $m^{\prime \prime}(z g ; m)=m^{\prime \prime}(g ; m)$. On reprend un par un les objets associés au début de cette section à $g$ et $m$ et qui servent à définir $V(g ; m)$ et $m^{\prime \prime}(g ; m)$, et on compare en suivant les définitions pas à pas ; on a :

$$
\text { - } F_{z g}=F_{g}
$$


$-e(z g)=e(g)$

- $\mathcal{G}(z g)=\mathcal{G}(g)$

- $J_{\mathcal{G}}(z g)=J_{\mathcal{G}}(g)$

- la valuation $\nu_{\mathcal{G}}$ est la même,

- $\mathcal{N}_{k}(z g)=\mathcal{N}_{k-\nu_{\mathcal{G}}(z)}(g)$

- $k_{0}(z g)=k_{0}(g)+\nu_{\mathcal{G}}(z)$

- $k_{1}(z g)=k_{1}(g) \operatorname{car} \nu_{\mathcal{G}}(z g)=\nu_{\mathcal{G}}(g)+\nu_{\mathcal{G}}(z)$

- $\bar{k}_{1}(z g)=\bar{k}_{1}(g)$,

- $A(z g)=A(g)$ car $\mathcal{N}_{k_{0}(z g)}(z g)=\mathcal{N}_{k_{0}(g)}(g)$ (même si $k_{0}(z g) \neq$ $\left.k_{0}(g)\right)$,

$-\Lambda(z g)=\Lambda(g)$

- $m^{\prime}(z g ; m)=m^{\prime}(g ; m)$

- $m^{\prime \prime}(z g ; m)=m^{\prime \prime}(g ; m)$.

La fonction à support compact $\phi: Z_{F}=F^{*} \rightarrow \mathbb{C}$ définie par

$$
\phi(z)=\omega(z) \Phi(f ; z g)
$$

est alors d'après le théorème ??.a invariante par le sous-groupe $(1+$ $\left.\pi^{m^{\prime}(g ; m)} \Lambda\right) \cap Z_{F}$ de $Z_{F}$. Soit $L$ un corps $m^{\prime \prime}(g ; m)$-proche de $F$, et soit $g^{\prime}$ un élément quelconque dans $\bar{\zeta}_{F L}^{m^{\prime \prime}}(V(g ; m))$. En appliquant le théorème ??.b, on trouve que la fonction $\phi^{\prime}: Z_{L}=L^{*} \rightarrow \mathbb{C}$ définie par

$$
\phi^{\prime}\left(z^{\prime}\right)=\bar{\zeta}_{F L}^{m^{\prime \prime}}(\omega)\left(z^{\prime}\right) \Phi\left(\bar{\zeta}_{F L}^{m^{\prime \prime}}(f) ; z^{\prime} g^{\prime}\right)
$$

a la même intégrale sur $Z_{L}$ que $\phi$ sur $Z_{F}$ car les deux intégrales sont des sommes finies de termes égaux deux à deux. Comme

$$
\int_{Z_{L}} \bar{\zeta}_{F L}^{m^{\prime \prime}}(\omega)\left(z^{\prime}\right) \Phi\left(\bar{\zeta}_{F L}^{m^{\prime \prime}}(f) ; z^{\prime} g^{\prime}\right) d z^{\prime}=\Phi\left(I_{\bar{\zeta}_{F L}^{m^{\prime \prime}}(\omega)}\left(\bar{\zeta}_{F L}^{m^{\prime \prime}}(f)\right) ; g^{\prime}\right)
$$

on trouve bien

$$
\Phi\left(I_{\omega}(f) ; g\right)=\Phi\left(I_{\bar{\zeta}_{F L}^{m^{\prime \prime}(\omega)}}\left(\bar{\zeta}_{F L}^{m^{\prime \prime}}(f)\right) ; g^{\prime}\right)
$$

Soit $\pi$ une représentation de carré intégrable de $G L_{n}(F)$ de caractère central (unitaire) $\omega$ et de niveau $m$.

Lemme 4.2. . a) Si $L$ est un corps m-proche de $F$, alors $\bar{\zeta}_{F L}^{m}(\pi)$ est une représentation de carré intégrable de $G L_{n}(L)$.

b) Soit $f_{\pi}$ un pseudocoefficient de $\pi$. Soit $h \in H\left(G L_{n}(F)\right)$ tel que $I_{\omega}(h)=f_{\pi}$. Soit $m^{\prime} \in \mathbb{N}$ tel qu'on ait $h \in H\left(G L_{n}(F) ; H_{F}^{m^{\prime}}\right)$. Alors $m^{\prime} \geq m$ et, si $L$ est un corps $m^{\prime}+1$-proche de $F$, alors $I_{\bar{\zeta}_{F L}^{m}(\omega)}\left(\bar{\zeta}_{F L}^{m}(h)\right)$ est un pseudocoefficient de $\bar{\zeta}_{F L}^{m}(\pi)$. 
Démonstration. a) Soit $\sigma$ une représentation (irréductible et de niveau $m$ ) se trouvant dans l'image par $\bar{\zeta}_{F L}^{m}$ de la classe d'équivalence de $\pi$. À la section précédente on a vu comment on construit $\sigma$ à partir de $\pi$. On emploie les mêmes notations. On a un isomorphisme d'espaces vectoriels

$$
f: V_{\pi}^{K_{F}^{m}} \simeq V_{\sigma}^{K_{L}^{m}} .
$$

L'isomorphisme $f$ induit un isomorphisme entre les duaux :

$$
f^{\prime}: V_{\pi}^{\prime} K_{F}^{m} \simeq V_{\sigma}^{\prime} K_{L}^{m}
$$

Soient $v \in V_{\pi}^{K_{F}^{m}} \backslash\{0\}$ et $v^{\prime} \in V_{\pi}^{\prime} K_{F}^{m} \backslash\{0\}$. Considérons le coefficient de $\pi$

$$
h_{\pi}: G L_{n}(F) \rightarrow \mathbb{C}
$$

défini par

$$
g \mapsto v^{\prime}(\pi(g)(v))
$$

C'est un coefficient non nul.

Pour tout $g \in G L_{n}(F)$, pour tout $g^{\prime} \in K_{F}^{m} g K_{F}^{m}$, on a

$$
\pi\left(g^{\prime}\right)=\operatorname{vol}\left(K_{F}^{m} g K_{F}^{m}\right)^{-1} \pi\left(\mathbf{1}_{K_{F}^{m} g K_{F}^{m}}\right) .
$$

La représentation $\pi$ étant de carré intégrable, $\left|h_{F}\right|^{2}$ est trivial sur $Z$ et intégrable sur $G L_{n}(F) / Z$. Exprimons cette propriété à partir de la décomposition

$$
G L_{n}(F)=\coprod_{A \in \mathcal{A}_{F}} \coprod_{(\tilde{B} ; \tilde{C}) \in \tilde{\mathbf{T}}_{F, m, A}} K_{F}^{m} \tilde{B} A \tilde{C}^{-1} K_{F}^{m}
$$

en étudiant l'action par multiplication de $Z$ là-dessus. Notons $\mathcal{A}_{F}^{0}$ le sous-ensemble de $\mathcal{A}_{F}$ formé de matrices $A=\operatorname{diag}\left(\pi_{F}^{a_{1}} ; \pi_{F}^{a_{2}} ; \ldots \pi_{F}^{a_{n}}\right)$ telles que $a_{1}=0$. Pour toute matrice $A \in \mathcal{A}_{F}^{0}$ notons $\mathcal{A}_{F}(A)$ l'ensemble de matrices obtenues à partir de $A$ par multiplication avec une puissance de $\pi_{F}$. C'est un sous-ensemble de $\mathcal{A}_{F}$. On a

$$
\mathcal{A}_{F}=\coprod_{A \in \mathcal{A}_{F}^{0}} \mathcal{A}_{F}(A) \text {. }
$$

Montrons que, pour tout $A \in \mathcal{A}_{F}^{0}$, l'ensemble $\coprod_{A \in \mathcal{A}_{F}(A)} \coprod_{(\tilde{B} ; \tilde{C}) \in \tilde{\mathbf{T}}_{F, m, A}} K_{F}^{m} \tilde{B} A \tilde{C}^{-1} K_{F}^{m}$ est stable sous l'action de $Z$ par multiplication et étudions cette action. Identifions $Z$ avec $F^{*}$. Alors, si $z$ est un élément de $Z, z$ s'écrit de façon unique $z=\pi_{F}^{\alpha} x$ où $x$ est un élément de $O_{F}^{*}$. D'autre part, on a un isomorphisme

$$
O_{F}^{*} /\left(1_{F}+P_{F}^{m}\right) \simeq\left(O_{F} / P_{F}^{m}\right)^{*}
$$

On peut ainsi décomposer l'action de $Z$ sur $\coprod_{A \in \mathcal{A}_{F}(A)} \coprod_{(\tilde{B} ; \tilde{C}) \in \tilde{\mathbf{T}}_{F, m, A}} K_{F}^{m} \tilde{B} A \tilde{C}^{-1} K_{F}^{m}$ puisque : 
- si $z=\pi_{F}^{\alpha}$, alors $z K_{F}^{m} \tilde{B} A \tilde{C}^{-1} K_{F}^{m}=K_{F}^{m} \tilde{B} A^{\prime} \tilde{C}^{-1} K_{F}^{m}$ où $A^{\prime}=z A \in$ $\mathcal{A}_{F}(A)$, et une simple vérification montre qu'on a dans ce cas $\tilde{\mathbf{T}}_{F, m, A}=$ $\tilde{\mathbf{T}}_{F, m, A^{\prime}}$

- si $z \in O_{F}^{*}$, alors :

- si $z \in 1_{F}+P_{F}^{m}$, on a $z K_{F}^{m} \tilde{B} A \tilde{C}^{-1} K_{F}^{m}=K_{F}^{m} \tilde{B} A \tilde{C}^{-1} K_{F}^{m}$, tandis que

- si $z \notin 1_{F}+P_{F}^{m}, z K_{F}^{m} \tilde{B} A \tilde{C}^{-1} K_{F}^{m}=K_{F}^{m} \tilde{B}^{\prime} A \tilde{C}^{\prime}-1 K_{F}^{m}$, où $\left(\tilde{B}^{\prime} ; \tilde{C}^{\prime}\right)$ est un élément de $\tilde{\mathbf{T}}_{F, m, A}$ différent de $(\tilde{B} ; \tilde{C})$ et qui ne dépend que de la classe de $z$ modulo $1_{F}+P_{F}^{m}$.

Finalement, dire que $\left|h_{\pi}\right|^{2}$ est intégrable sur $G L_{n}(F) / Z$ revient à dire que la somme

$$
\begin{gathered}
\sum_{A \in \mathcal{A}_{F}^{0}}\left(\sum_{(\tilde{B} ; \tilde{C}) \in \tilde{\mathbf{T}}_{F, m, A}}\left(\operatorname{card}\left(\left(O_{F} / P_{F}^{m}\right)^{*}\right)\right)^{-1}\right. \\
\left.\operatorname{vol}\left(K_{F}^{m} \tilde{B} A \tilde{C}^{-1} K_{F}^{m} ; d g\right)\left(\operatorname{vol}\left(1_{F}+P_{F}^{m} ; d z\right)\right)^{-1}\left|h_{\pi}\left(\tilde{B} A \tilde{C}^{-1}\right)\right|^{2}\right)
\end{gathered}
$$

est convergente, c.-̀̀-d., en tenant compte de $\operatorname{vol}\left(1_{F}+P_{F}^{m} ; d z\right) \operatorname{card}\left(\left(O_{F} / P_{F}^{m}\right)^{*}\right)=$ $\operatorname{vol}\left(O_{F}^{*} ; d z\right)=1$, que la somme

$$
\sum_{A \in \mathcal{A}_{F}^{0}}\left(\sum_{(\tilde{B} ; \tilde{C}) \in \tilde{\mathbf{T}}_{F, m, \boldsymbol{A}}} \operatorname{vol}\left(K_{F}^{m} \tilde{B} A \tilde{C}^{-1} K_{F}^{m} ; d g\right)\left|h_{\pi}\left(\tilde{B} A \tilde{C}^{-1}\right)\right|^{2}\right)
$$

est convergente. Maintenant, $f(v)$ est un élément de $V_{\sigma}^{K_{L}^{m}} \backslash\{0\}$ et $f^{\prime}\left(v^{\prime}\right)$ est un élément de $V_{\sigma}^{\prime} K_{L}^{m} \backslash\{0\}$, donc l'application

$$
h_{\sigma}: G L_{n}(L) \rightarrow \mathbb{C}
$$

définie par

$$
g \mapsto f\left(v^{\prime}\right)(\sigma(g)(f(v)))
$$

est un coefficient de $\sigma$.

Pour tout $g \in G L_{n}(L)$, pour tout $g^{\prime} \in K_{L}^{m} g K_{L}^{m}$ égal à

$$
\sigma\left(g^{\prime}\right)=\operatorname{vol}\left(K_{L}^{m} g K_{L}^{m}\right)^{-1} \sigma\left(\mathbf{1}_{K_{L}^{m} g K_{L}^{m}}\right) .
$$

Donc $h_{\sigma}$ est de carré intégrable modulo le centre sur $G L_{n}(L)$ si et seulement si la somme

$$
\sum_{A \in \mathcal{A}_{F}^{0}}\left(\sum_{(\tilde{B} ; \tilde{C}) \in \tilde{\mathbf{T}}_{F, m, A}} \operatorname{vol}\left(K_{L}^{m} \tilde{B} \zeta_{F L}(A) \tilde{C}^{-1} K_{L}^{m} ; d g\right)\left|h_{\sigma}\left(\tilde{B} \zeta_{F L}(A) \tilde{C}^{-1}\right)\right|^{2}\right)
$$

est convergente, où on a tenu compte du fait que $\zeta_{F L}^{m}$ réalise une bijection de $\mathcal{A}_{F}^{0}$ sur $\mathcal{A}_{L}^{0}$. Mais cette somme correspond terme pour terme à la somme (??) puisque

- les volumes des sous-ensembles de $G L_{n}(F)$ et $G L_{n}(L)$ qui se correspondent sont égaux pour les mesures fixées sur $G L_{n}(F)$ et $G L_{n}(L)$, 
- par construction de l'isomorphisme $f$, pour tout $v \in V_{\pi}^{K_{F}^{m}}$ on a

$$
f\left(\pi\left(\mathbf{1}_{K_{F}^{m} g K_{F}^{m}}\right)(v)\right)=\sigma\left(\mathbf{1}_{K_{L}^{m} g K_{L}^{m}}\right)(f(v)) .
$$

On vient de montrer qu'un coefficient non nul de $\sigma$ est de carré intégrable sur $G L_{n}(L) / Z$. Donc $\sigma$ est de carré intégrable.

b) Évidemment $m^{\prime} \geq m$, puisque $\operatorname{tr} \pi(h)=\operatorname{tr} \pi\left(f_{\pi}\right) \neq 0$. Montrons que $I_{\bar{\zeta}_{F L}^{m}(\omega)}\left(\bar{\zeta}_{F L}^{m}(h)\right)$ vérifie la définition d'un pseudocoefficient de $\bar{\zeta}_{F L}^{m}(\pi)$ : d'une part

$\operatorname{tr} \bar{\zeta}_{F L}^{m}(\pi)\left(I_{\bar{\zeta}_{F L}^{m}(\omega)}\left(\bar{\zeta}_{F L}^{m}(h)\right)\right)=\operatorname{tr} \bar{\zeta}_{F L}^{m}(\pi)\left(\bar{\zeta}_{F L}^{m}(h)\right)=\operatorname{tr} \pi(h)=\operatorname{tr} \pi\left(f_{\pi}\right)=1 ;$

d'autre part, si $\sigma^{\prime}$ est une représentation irréductible essentiellement tempérée de $G L_{n}(L)$ de caractère central $\bar{\zeta}_{F L}^{m}(\omega)$ mais non équivalente à $\bar{\zeta}_{F L}^{m}(\pi)$, alors ou bien $\sigma^{\prime}$ est de niveau supérieur strictement à $m^{\prime}$ alors que $\bar{\zeta}_{F L}^{m}(h)$ est invariante par $K_{L}^{m^{\prime}}$ et on a de ce fait

$$
\operatorname{tr} \sigma^{\prime}\left(\bar{\zeta}_{F L}^{m}(h)\right)=0,
$$

ou bien le niveau de $\sigma^{\prime}$ est inférieur ou égal à $m^{\prime}$. Alors $\sigma^{\prime}$ qui est essentiellement tempérée irréductible est en particulier générique irréductible ([Ze], th.9.7), donc image par $\bar{\zeta}_{F L}^{m}$ d'une représentation générique irréductible $\sigma$ de $G L_{n}(F)$ (prop. ??), de caractère central $\omega$, mais non équivalente à $\pi$, et on a

$$
\operatorname{tr} \sigma^{\prime}\left(\bar{\zeta}_{F L}^{m}(h)\right)=\operatorname{tr} \sigma(h)=\operatorname{tr} \sigma\left(f_{\pi}\right) .
$$

Mais $\sigma$, étant générique, est ou bien une représentation essentiellement de carré intégrable, ou bien une représentation induite à partir d'un sous-groupe parabolique propre ([Ze], th.9.7). On a dans le premier cas $\operatorname{tr} \sigma\left(f_{\pi}\right)=0$ par la définition d'un pseudocoefficient de $\pi$ et encore $\operatorname{tr} \sigma\left(f_{\pi}\right)=0$ dans le deuxième cas par le théorème ??.b.

Montrons maintenant que $G L_{n}(F)$ a la propriété $\mathbf{P}$ :

Théorème 4.3. . L'intégrale orbitale de $f_{\pi}$ vérifie :

(i) $\Phi\left(f_{\pi} ; g\right)=0$ si $g$ est semisimple régulier non elliptique,

(ii) $\Phi\left(f_{\pi} ; g\right)=\overline{\chi_{\pi}(g)}$ si $g$ est elliptique régulier.

Démonstration. (i) C'est le point c) du théorème ??.

(ii) Considérons une fonction $h \in H\left(G L_{n}(F)\right)$ telle qu'on ait $I_{\omega}(h)=$ $f_{\pi}$. Supposons que $h \in H\left(G L_{n}(F) ; K_{F}^{m^{\prime}}\right)$. Soit $g$ un élément elliptique régulier. Soit $W$ un voisinage de $g$ sur lequel $\chi_{\pi}$ est constant et 
soit $m$ un entier suffisamment grand pour qu'on ait $V(g ; m) \subset W$ et que le lemme précédent soit vérifié (i.e. si $L$ est un corps local non archimédien de caractéristique nulle $m$-proche de $F$, alors $\bar{\zeta}_{F L}^{m}(\pi)$ est une représentation essentiellement de carré intégrable de $G L_{n}(L)$ et $I_{\bar{\zeta}_{F L}^{m}(\omega)}\left(\bar{\zeta}_{F L}^{m}(h)\right)$ est un pseudocoefficient de $\left.\bar{\zeta}_{F L}^{m}(\pi)\right)$. Soit $L$ un corps de caractéristique nulle, $m^{\prime \prime}(g ; m)$-proche de $F$. On a dans cette situation :

1) $\chi_{\pi}$ est constant sur $V(g ; m)$ égal à $\chi_{\pi}(g)$, car $V(g ; m) \subset W$,

2) $\Phi\left(I_{\omega}(h) ;\right)$ est constante sur $V(g ; m)$ égale à $\Phi\left(I_{\omega}(h) ; g\right)$ (th.??) et

3) $\Phi\left(I_{\bar{\zeta}_{F L}^{m}(\omega)}\left(\bar{\zeta}_{F L}^{m^{\prime \prime}}(h)\right) ;\right.$ ) est constante sur $\bar{\zeta}_{F L}^{m^{\prime \prime}}(V(g ; m))$ égale à $\Phi\left(I_{\omega}(h) ; g\right)$ (th.??).

Or, $I_{\omega}(h)=f_{\pi}$. Le corps $L$ étant de caractéristique nulle, $G L_{n}(L)$ a la propriété $\mathbf{P}$. Du lemme ??.b on en déduit alors que : $\chi_{\bar{\zeta}_{F L}^{m}}^{m^{\prime \prime}(\pi)}$ est constant sur $\bar{\zeta}_{F L}^{m^{\prime \prime}}(V(g ; m))$ égal à $\overline{\Phi\left(I_{\bar{\zeta}_{F L}^{m^{\prime \prime}}(\omega)}\left(\bar{\zeta}_{F L}^{m^{\prime \prime}}(h)\right) ; g\right)}$. Finalement, en appliquant le point 3$)$ ci-dessus, on trouve

4) $\chi_{\bar{\zeta}_{F L}^{m^{\prime \prime}(\pi)}}$ est constant sur $\bar{\zeta}_{F L}^{m^{\prime \prime}}(V(g ; m))$ égal à $\overline{\Phi\left(f_{\pi} ; g\right)}$.

En notant $I$ la fonction caractéristique de $V(g ; m)$, de 1$)$ on a

$$
\operatorname{tr} \pi(I)=\operatorname{vol}(V(g ; m)) \chi_{\pi}(g) .
$$

D'autre part $\bar{\zeta}_{F L}^{m^{\prime \prime}}(I)$ est bien définie et est la fonction caractéristique de $\bar{\zeta}_{F L}^{m^{\prime \prime}}(V(g ; m))$ (par définition même de cette dernière) et alors de 4) on en déduit que

$$
\operatorname{tr}\left(\bar{\zeta}_{F L}^{m^{\prime \prime}}(\pi)\right)\left(\bar{\zeta}_{F L}^{m^{\prime \prime}}(I)\right)=\operatorname{vol}\left(\bar{\zeta}_{F L}^{m^{\prime \prime}}(V(g ; m))\right) \overline{\Phi\left(f_{\pi} ; g\right)}
$$

Comme on a

$$
\operatorname{vol}\left(\bar{\zeta}_{F L}^{m^{\prime \prime}}(V(g ; m))\right)=\operatorname{vol}(V(g ; m))
$$

et qu'on sait que

$$
\operatorname{tr}\left(\bar{\zeta}_{F L}^{m^{\prime \prime}}(\pi)\right)\left(\bar{\zeta}_{F L}^{m^{\prime \prime}}(I)\right)=\operatorname{tr} \pi(I)
$$

on trouve bien

$$
\chi_{\pi}(g)=\overline{\Phi\left(f_{\pi} ; g\right)}
$$

Donc $G L_{n}(F)$ a la propriété $\mathbf{P}$. Comme sur $G L_{n}(F)$ il y a intégrabilité locale du caractère de toute représentation lisse irréductible ([Le2]), on en déduit par la proposition ?? le 
Théorème 4.4. . (Orthogonalité des caractères pour $G L_{n}$ ) L'ensemble des fonctions caractères associées aux classes d'équivalence des représentations de carré intégrable de $G L_{n}(F)$ de caractère central $\omega$ fixé forme un système orthonormal pour l'espace préhilbertien $L^{2}\left(G L_{n}(F)_{e} ; \omega ;<;>_{e}\right.$ ) .

\section{Applications}

Soient $F$ un corps local non archimédien de caractéristique quelconque et $D$ une algèbre à division centrale de dimension finie sur $F$. Soit $D^{*}$ le groupe des éléments inversibles de $D$. La dimension de $D$ en tant qu'espace vectoriel sur $F$ est un carré $n^{2}$. On pose $G=G L_{n}(F)$. On fixe des mesures de Haar sur $G$ et $D^{*}$. Si $g$ est un élément de $G$ et $g^{\prime}$ un élément de $D^{*}$ on écrit $g \leftrightarrow g^{\prime}$ si $g$ et $g^{\prime}$ sont réguliers et ont le même polynôme caractéristique. On dit alors que $g$ et $g^{\prime}$ se correspondent. Ça revient à dire que $g$ est elliptique régulier et a le même polynôme caractéristique que $g^{\prime}$. Il existe des isomorphismes canoniques $Z_{G} \simeq F^{*}$ et $Z_{D^{*}} \simeq F^{*}$ et donc un isomorphisme canonique $Z_{G} \simeq Z_{D^{*}}$. On note ces deux groupes indistinctement $Z$. On fixe une mesure de Haar $d z$ sur $Z$. Sur tout tore maximal elliptique $T$ de $G$ ou de $D^{*}$ on considère la mesure de Haar $d t$ telle que $T / Z$ ait volume 1 pour la mesure quotient $d \bar{t}=d t / d z$. Sur les tores non elliptiques de $G$ on fixe des mesures de Haar quelconques.

Soit $\omega$ un caractère unitaire de $Z$. Il y a un isomorphisme d'espaces vectoriels :

défini par

$$
i: L^{0}\left(G_{e} ; \omega\right) \simeq L^{0}\left(D_{e}^{*} ; \omega\right)
$$

$$
i(f)\left(g^{\prime}\right)=f(g) \quad \text { si } g \leftrightarrow g^{\prime}
$$

pour tout $f \in L^{0}\left(G_{e} ; \omega\right)$. Maintenant, si $g \leftrightarrow g^{\prime}$, alors il y a un unique isomorphisme de $F$-algèbres (qui dans ce cas précis sont des corps) $F[g] \simeq F\left[g^{\prime}\right]$, qui envoie $g$ sur $g^{\prime}$. On a donc un isomorphisme de groupes multiplicatifs $F[g]^{*} \simeq F\left[g^{\prime}\right]^{*}$. Mais $F[g]^{*}$ n'est autre que le groupe des points du tore maximal elliptique $Z(g)$ qui contient $g$ et $F\left[g^{\prime}\right]^{*}$ n'est autre que le groupe des points d'un tore maximal elliptique $Z\left(g^{\prime}\right)$ qui contient $g^{\prime}$. Toutes ces considérations impliquent que, si on choisit un ensemble de représentants $\mathcal{T}_{e}$ des classes de conjugaison de tores maximaux elliptiques de $G$ et un ensemble de représentants $\mathcal{T}^{\prime}{ }_{e}$ des classes de conjugaison de tores maximaux elliptiques de $D^{*}$, il y a une bijection

$$
j: \mathcal{T}_{e} \rightarrow \mathcal{T}_{e}^{\prime}
$$

qui est caractérisée par $T \simeq j(T)$. 
Soit $T \in \mathcal{T}_{e}$. Notons $j_{T}$ un isomorphisme de $T$ sur $j(T)$. Étant un morphisme de groupes, $j_{T}$ transforme la mesure de $\mathrm{T}$ en une mesure de Haar de $j(T)$, donc en un multiple complexe de la mesure qu'on avait choisi sur $j(T)$. Mais la restriction de l'application $j_{T}$ à $Z$ est l'identité, donc, vu le choix des mesures sur $T$ et $j(T)$, on en déduit que $j_{T}$ préserve la mesure. Alors la restriction de l'isomorphisme $i$ à $L^{2}\left(G_{e} ; \omega\right)$ induit un isomorphisme d'espaces préhilbertiens de $L^{2}\left(G_{e} ; \omega\right)$ sur $L^{2}\left(D_{e}^{*} ; \omega\right)$. D'une façon plus explicite, on a :

$$
<i(f) ; i(h)>_{e}=<f ; h>_{e} \quad \forall f, h \in L^{2}\left(G_{e} ; \omega\right) .
$$

Si $f \in H(G)$ et $f^{\prime} \in H\left(D^{*}\right)$ on dit que $f$ et $f^{\prime}$ se correspondent si leurs intégrales orbitales ont la même valeur sur des éléments de $G$ et de $D^{*}$ qui se correspondent et que les intégrales orbitales de $f$ sont nulles sur les éléments semisimples réguliers non elliptiques de $G$. On écrit alors $f \leftrightarrow f^{\prime}$. On dit aussi que $f$ se transfère ou que $f^{\prime}$ se transfère. Si $\omega$ est un caractère de $Z$ la définition plus haut vaut aussi pour $f \in H(G ; \omega)$ et $f^{\prime} \in H\left(D^{*} ; \omega\right)$.

On rappelle que $E^{2}(G)$ est l'ensemble des classes d'équivalence de représentations essentiellement de carré intégrable de $G$ et que $E\left(D^{*}\right)$ est l'ensemble des classes d'équivalence de représentations irréductibles de $D^{*}$ (qui sont toutes essentiellement de carré intégrable et même cuspidales, puisque $D^{*}$ est compact modulo le centre).

Théorème 5.1. . Il existe une unique bijection :

$$
\mathrm{C}: E^{2}(G) \rightarrow E\left(D^{*}\right)
$$

telle que pour tout $\pi \in E^{2}(G)$ on ait

$$
\chi_{\pi}(g)=(-1)^{n-1} \chi_{\mathbf{C}(\pi)}\left(g^{\prime}\right) \quad \text { pour tout } g \leftrightarrow g^{\prime} .
$$

Les représentations $\pi$ et $\mathbf{C}(\pi)$ ont le même caractère central.

$L$ 'application $\mathbf{C}$ commute à la tensorisation avec les caractères.

On a aussi $\operatorname{tr} \pi(f)=(-1)^{n-1} \operatorname{tr} \mathbf{C}(\pi)\left(f^{\prime}\right)$ pour tout $f \leftrightarrow f^{\prime}$.

Démonstration. Rogawski a donné une démonstration de ce résultat dans le cas où $F$ est de caractéristique nulle dans [Ro]. La démonstration de Rogawski a été reprise et simplifiée dans [DKV] et finalement Flicker a donné une preuve encore plus simple dans [Fl]. La démonstration de Flicker s'applique maintenant mot pour mot en caractéristique non nulle, puisque le seul résultat qui lui manquait était l'orthogonalité des caractères sur $G$ (th.??, plus haut). La relation entre les distributions traces est impliquée par l'intégrabilité locale des caractères à la fois sur 
$G L_{n}(F)([\mathrm{Le} 2])$ et sur $D^{*}$ (parce qu'il est compact modulo le centre).

Corollaire 5.2. . Soit $\omega$ un caractère unitaire de $Z$. L'ensemble des restrictions à $G_{e}$ des caractères de toutes les représentations de carré intégrable de $G$ de caractère central $\omega$ est un système orthonormal complet de l'espace $L^{2}\left(G_{e} ; \omega\right)$ (ou de son complété hilbertien).

Démonstration. C'est vrai pour $D^{*}$ et $L^{2}\left(D^{*} ; \omega\right)$ parce que $D^{*}$ est compact modulo $Z$. Par la correspondance et via l'isomorphisme $i$ défini plus haut, ce résultat est vrai aussi pour $G$.

Théorème 5.3. . Soit $\omega$ un caractère de $Z$.

(a) Pour tout $f \in H(G)$ dont l'intégrale orbitale est nulle sur $G^{s r} \backslash G_{e}$ il existe une fonction $f^{\prime} \in H\left(D^{*}\right)$ telle que $f \leftrightarrow f^{\prime}$ et pour tout $f \in$ $H(G ; \omega)$ dont l'intégrale orbitale est nulle sur $G^{s r} \backslash G_{e}$ il existe un $f^{\prime} \in$ $H\left(D^{*} ; \omega\right)$ tel que $f \leftrightarrow f^{\prime}$.

(b) Pour tout $f \in H\left(D^{*}\right)$ il existe un $f^{\prime} \in H(G)$ tel que $f \leftrightarrow f^{\prime}$ et pour tout $f^{\prime} \in H\left(D^{*} ; \omega\right)$ il existe un $f \in H(G ; \omega)$ tel que $f \leftrightarrow f^{\prime}$.

Démonstration. On a montré (th.??) que $G$ avait la propriété $\mathbf{P}$. Le groupe $D^{*}$ a aussi la propriété $\mathbf{P}$ parce qu'il est compact modulo le centre. Le théorème de correspondance plus haut implique alors qu'un pseudocoefficient d'une représentation $\pi$ essentiellement de carré intégrable de $G$ correspond à un (pseudo)coeffici-

ent de $\mathbf{C}(\pi)$ multiplié par $(-1)^{n-1}$ (le passage des représentations de carré intégrable aux représentations essentiellement de carré intégrable se fait en tordant par des caractères). Donc les pseudocoefficients se transfèrent.

Soient $\omega$ un caractère de $Z$ et $f$ une fonction dans $H\left(D^{*} ; \omega\right)$. Si on pose

$$
h=f-\sum_{\pi} \operatorname{tr} \pi(f) f_{\pi},
$$

où la somme porte sur le nombre fini de classes de représentations $\pi$ de $D^{*}$ de caractère central $\omega$ dont la trace ne s'annule pas sur $f$ et, pour chaque tel $\pi, f_{\pi}$ désigne un pseudocoefficient, on trouve que pour toute représentation $\pi$ de $D^{*}$ de caractère central $\omega, \operatorname{tr} \pi(h)=0$; ce qui implique que l'intégrale orbitale de $h$ s'annule sur les éléments réguliers de $D^{*}$ ([DKV], th.A.2.a, variante 2). On en déduit que la famille des intégrales orbitales des (pseudo)coefficients des représentations de $D^{*}$ de caractère central $\omega$ est une base (algébrique) pour le sous-espace de $L^{0}\left(D^{*} ; \omega\right)$ formé par les intégrales orbitales des fonctions de $H\left(D^{*} ; \omega\right)$. 
Prenons cette fois une fonction $f \in H(G ; \omega)$ dont l'intégrale orbitale s'annule sur les éléments semisimples réguliers non elliptiques et considérons la fonction

$$
h=f-\sum_{\pi} \operatorname{tr} \pi(f) f_{\pi},
$$

où la somme porte sur le nombre fini de classes de représentations essentiellement de carré intégrable de $G$ de caractère central $\omega$ dont la trace ne s'annule pas sur $f$. D'après la classification de Zelevinski, les classes d'équivalence des représentations qui sont des induites (strictes on non) de représentations essentiellement de carré intégrable forment une base de $G r o t(G)$. Décomposons une représentation lisse irréductible $\pi$ de $G$ sur cette base. Si le caractère central de $\pi$ est $\omega$, le caractère central de ces représentations essentiellement de carré intégrable ou induites de représentations essentiellement de carré intégrable est aussi $\omega$, par indépendance linéaire des caractères sur $Z=F^{*}$. Mais les traces des représentations essentiellement de carré intégrable s'annulent sur $h$ (par simple calcul) et les traces des représentations strictement induites de caractère central $\omega$ s'annulent sur $h$ parce que les intégrales orbitales de $h$ sont nulles sur les éléments semisimples réguliers non elliptiques et parce que les caractères sont localement intégrables sur $G$ ([Le2]). On en déduit que la trace de toute représentation lisse irréductible de $G$ de caractère central $\omega$ est nulle sur $h$. Donc l'intégrale orbitale de $h$ est nulle sur tout élément semisimple régulier de $G$ ([DKV], th.A.2.a, variante 2). On obtient (cette fois sur $G$ ) : les intégrales orbitales des pseudocoefficients des représentations essentiellement de carré intégrable de caractère central $\omega$ forment une base du sous-espace de $L^{0}(G ; \omega)$ formé par les intégrales orbitales des fonctions de $H(G ; \omega)$ dont les intégrales orbitales s'annulent sur les éléments semisimples réguliers non elliptiques.

D'après ce qu'on vient de voir et le fait que les pseudocoefficients des représentations essentiellement de carré intégrable se transfèrent dans les deux sens on obtient les moitiés des points a) et b) qui concernent les fonctions dans $H(G ; \omega)$ et dans $H\left(D^{*} ; \omega\right)$.

Pour obtenir la variante du transfert pour les fonctions à support compact, i.e. a) et b) pour $H(G)$ et $H\left(D^{*}\right)$, on utilise le théorème de Paley-Wiener : soit $f^{\prime}$ une fonction dans $H\left(D^{*}\right)$. Il existe alors une fonction $f$ dans $H(G)$ telle que pour toute représentation $\pi$ essentiellement de carré intégrable de $G$ on ait

1) $\operatorname{tr} \pi(f)=\operatorname{tr}[\mathbf{C}(\pi)]\left(f^{\prime}\right)$ et

2) $\operatorname{tr} \pi(f)=0$ pour toute représentation induite sur $G$ (les conditions de Paley-Wiener ([BDK] 1.2) sont très faciles à vérifier sur $\left.G L_{n}\right)$. 
La condition 2) implique que l'intégrale orbitale de $f$ est nulle sur les éléments semisimples réguliers non elliptiques de $G$.

La condition 1) implique que pour tout caractère $\omega$ de $Z$ et toute représentation essentiellement de carré intégrable $\pi$ de $G$ de caractère central $\omega$ on a $\operatorname{tr} \pi\left(I_{\omega}(f)\right)=\operatorname{tr} \mathbf{C}(\pi)\left(I_{\omega}\left(f^{\prime}\right)\right)$. La démonstration plus haut implique alors que les intégrales orbitales de $I_{\omega}(f)$ et de $I_{\omega}\left(f^{\prime}\right)$ sont égales sur les éléments elliptiques réguliers qui se correspondent. En se rappelant le lien entre l'intégrale orbitale de $f$ et celle de $I_{\omega}(f)$ on trouve que les intégrales orbitales $\Phi(f ;)$ et $\Phi\left(f^{\prime} ;\right)$ de $f$ et $f^{\prime}$ respectivement ont la propriété suivante : pour tout élément elliptique régulier $g$ de $G$, pour tout caractère $\omega$ de $Z$,

$$
\int_{Z}\left(\Phi(f ; z g)-\Phi\left(f^{\prime} ; z g^{\prime}\right)\right) \omega(z) d z=0
$$

On en déduit par le th.4.4, chap.2, [Bou], que $\Phi(f ; g)=\Phi\left(f ; g^{\prime}\right)$ pour tout $g \leftrightarrow g^{\prime}$ et donc $f \leftrightarrow f^{\prime}$ et $f^{\prime}$ se transfère. Le transfert dans l'autre sens se fait de la même façon.

\section{Bibliographie}

[Ba] A.I.Badulescu, thèse, Univ.Paris Sud, 1999.

[Bou] Bourbaki, Théories spectrales, Chap.1-2, Hermann, Paris

[Cl] L.Clozel, Invariant harmonic analysis on the Schwarz space of a reductive $p$-adic group, Proc.Bowdoin Conf.1989, Progress in Math.Vol.101, Birkhäuser, Boston, 1991, 101-102

[DKV] P.Deligne, D.Kazhdan, M.-F.Vignéras, Représentations des algèbres centrales simples $p$-adiques, Représentations des groupes réductifs sur un corps local, Hermann, Paris 1984.

[Fl] Y.Flicker, Transfer of Orbital Integrals and Division Algebras, J.Ramanujan Math.Soc. 5 (1990), 107-122.

[H-C1] Harish-Chandra, Admissible invariant distributions on reductive p-adic groups, Queen's Papers in Pure and Applied Mathematics 48 (1978), 281-347. 
[H-C2] Harish-Chandra, A submersion principle and its applications, Proc.Indian Acad.Sc. 90 (1981), 95-102.

[He] G.Henniart, On the Langlands conjecture for $G L(n)$ : the cyclic case, Annals of Math. 123 (1986), 145-203.

[La] G.Laumon, Cohomology with compact support of Drinfeld modular varieties, Cambridge Univ.Press, 1997.

[Le1] B.Lemaire, thèse, Univ.Paris Sud, 1994.

[Le2] B.Lemaire, Intégrabilité locale des caractères-distributions de $G L_{N}(F)$ où $F$ est un corps local non-archimédien de caractéristique quelconque, Compos. Math. 100 (1996), 41-75.

[Le3] B.Lemaire, Intégrales orbitales sur $G L(N)$ et corps locaux proches, Ann.Inst. Fourier 46 (1996), 1027-1056.

[Ro] J.Rogawski, Representations of $G L(n)$ and division algebras over a p-adic field, Duke Math. J. 50 (1983), 161-201.

[Ze] A.Zelevinski, Induced representations of reductive $p$-adic groups II, Ann. Sci. ENS 13 (1980), 165-210. 